\title{
ON THE DENSITY OF THE DISTRIBUTION OF $p$-STABLE SEMINORMS, $0<p<1$
}

\author{
MACIEJ LEWANDOWSKI AND TOMASZ ŻAK
}

\begin{abstract}
Let $\mu$ be a symmetric $p$-stable measure, $0<p<1$, on a locally convex separable linear metric space $E$ and let $q$ be a lower semicontinuous seminorm on $E$. It is known that $F(t)=\mu\{x: q(x)<t\}$ is absolutely continuous with respect to the Lebesgue measure. We prove an explicit formula for the density $F^{\prime}(t)$ and give an asymptotic estimate of it at infinity.
\end{abstract}

1. Let $X$ be a symmetric Gaussian random element with values in a locally convex metric linear space $E$ with a measurable seminorm $q$. Then the distribution function $F(t)=P\{q(X)<t\}$ is absolutely continuous apart from one possible jump $[3,4]$. In these papers some properties of the density $F^{\prime}(t)$ were also investigated.

Recently it has been shown $[2,6]$ that if $X$ is a symmetric $p$-stable random element with values in a linear metric separable space $E$ and $q$ is a lower semicontinuous seminorm on $E$ such that $P\{q(X)<\infty\}=1$, then the distribution function $F(t)=P\{q(X)<t\}$ is absolutely continuous (apart from one possible jump for $1 \leq p<2)$.

In [5] Ryznar gave an estimate of the density $F^{\prime}(t)$ for $p<1$ (see Lemma 1 below).

The aim of this paper is to prove that if $p<1$, then $F^{\prime}(t)$ exists for all $t>0$ and is continuous. The asymptotic behavior of $F^{\prime}(t)$ is also given.

2. In this paper $E$ will stand for a locally convex separable linear metric space. By $q$ we denote a measurable seminorm on $E$, i.e. a measurable function $q: E \rightarrow$ $[0, \infty]$ such that $q(x+y) \leq q(x)+q(y)$ and $q(\alpha x)=|\alpha| q(x)$ for all $x, y \in E$ and $\alpha \in R$. We will always assume in the sequel that $q$ is lower semicontinuous.

A probability measure $\mu$ on $E$ is called strictly $p$-stable if for every two independent random vectors $X$ and $Y$ with distributions $\mu$ and for every $\alpha, \beta>0$, $\alpha^{p}+\beta^{p}=1$, the distribution $\alpha X+\beta Y$ is equal to $\mu$. We will assume in addition that $\mu$ is symmetric.

If $\mu$ is a symmetric $p$-stable measure on $E$, then there exists a symmetric $\sigma$-finite measure $\nu$ on $E, \nu\left(U^{c}\right)<\infty$ for every open neighborhood $U$ of the orgin, and such that $\mu=\lim \exp \left(\nu \mid U_{n}^{c}\right)$ for $U_{n} \searrow\{0\}$. The measure $\nu$ is called the Lévy measure of $\mu$ and has the following property: $\nu(s A)=s^{-p} \nu(A)$ for every Borel set $A$. If $q<\infty \mu$-a.s., then there exists a finite symmetric measure $\sigma$ on $S_{1}=\{x: q(x)=1\}$ such that if $r(x)=q(x)$ and $s(x)=x / q(x)$ then

$$
\left.\nu\right|_{\{q>\varepsilon\}}\{x: q(x+y) \in A\}=\int_{S_{1}} \int_{\varepsilon}^{\infty} \mathbf{1}_{A}(q(r s+y)) \frac{d r}{r^{1+p}} \sigma(d s)
$$

Received by the editors May 20, 1985 and, in revised form, April 2, 1986. 1980 Mathematics Subject Classification (1985 Revision). Primary 60B05; Secondary 60E07. Key words and phrases. Stable measures, seminorms, density. 
for every $\varepsilon>0$ and Borel set $A$. We call $\sigma$ the spectral measure for $\mu$ (see [2] for details).

Observe that if $\mu_{t}$ is a distribution of $t^{1 / p} X$, where $X$ has the distribution $\mu$, then $\left(\mu_{t}\right)_{t>0}$ is a continuous convolution semigroup (i.e. $\mu_{t} * \mu_{s}=\mu_{t+s}$ and $\mu_{t} \Rightarrow \delta_{0}$ weakly) of $p$-stable measures and $\mu_{1}=\mu$.

It is known (see [2 or 6]) that if $\mu$ is a symmetric $p$-stable measure on $E, 0<$ $p<2$, then $F(t)=\mu\{x: q(x)<t\}$ is an absolutely continuous function (apart from one possible jump for $1 \leq p<2)$. When $0<p<1$ the density $F^{\prime}(t)$, as we show has additional regularity properties.

The first result about behavior of $F^{\prime}(t)$ was obtained by Ryznar [5]:

LEMMA 1. If $\mu$ is a symmetric $p$-stable measure, $0<p<1$, on a locally convex separable metric linear space $E$ and $q$ is a lower semicontinuous seminorm on $E$, then the density $F^{\prime}(t)$ (which exists for almost all $t>0$ ) has the following property: $\forall \varepsilon>0 \exists M_{\varepsilon}>0 F^{\prime}(t)<M_{\varepsilon}$, for all $t>\varepsilon$ for which $F^{\prime}(t)$ exists.

We also need the following fact due to M. Ryznar (unpublished). Denote by $\nu$ the Lévy measure of a $p$-stable measure $\mu$.

LEMMA 2. Let $\mu$ be a symmetric p-stable $0<p<1$, measure on a separable metric linear space $E$ and let $q$ be a lower semicontinuous seminorm on $E$. Then

$$
\lim _{t \rightarrow 0+} \frac{1}{t} \mu_{t}\{x: q(x)>\varepsilon\}=\nu\{x: q(x)>\varepsilon\}
$$

whenever $\nu\{x: q(x)=\varepsilon\}=0$.

PROOF. It is well known that for every open neighborhood $U$ of the origin such that $\nu(\partial U)=0$ the measures $\left(t^{-1} \mu_{t} \mid U^{c}\right)$ converge weakly to $\left(\nu \mid U^{c}\right)$. Hence, if $q$ is a continuous seminorm, then $\left(t^{-1} \mu_{t}\right) \mid\{x: q(x)>\varepsilon\}$ converges weakly to $\left.\nu\right|_{\{x: q(x)>\varepsilon\}}$ whenever $\nu\{x: q(x)=\varepsilon\}=0$.

If $q$ is lower semicontinuous, then there exists a sequence $\left(q_{n}\right)_{n=1}^{\infty}$ of continuous seminorms such that $q_{n} \nearrow q$. Let $\varepsilon>0$ be such that $\nu\{x: q(x)=\varepsilon\}=0$. Then

$$
\liminf _{t \rightarrow 0+} \frac{1}{t} \mu_{t}\{x: q(x)>\varepsilon\} \geq \nu\{x: q(x)>\varepsilon\}
$$

because the set $\{x: q(x)>\varepsilon\}$ is open. We show that

$$
\limsup _{t \rightarrow 0+} \frac{1}{t} \mu_{t}\{x: q(x)>\varepsilon\} \leq \nu\{x: q(x)>\varepsilon\} .
$$

For every $n \in N$ and $\varepsilon>0$ such that $\nu\{x: q(x)=\varepsilon\}=0$ we have

$$
\lim _{t \rightarrow 0+} \frac{1}{t} \mu_{t}\left\{x: q_{n}(x)>\varepsilon\right\}=\nu\left\{x: q_{n}(x)>\varepsilon\right\}
$$

and

$$
\lim _{n} \nu\left\{x: q_{n}(x)>\varepsilon\right\}=\nu\{x: q(x)>\varepsilon\} .
$$

Let us denote by $\left(X_{i}\right)_{i=1}^{\infty}$ a sequence of independent random elements with the distribution $\mu$. We obtain the following inequality:

$$
P\left\{q_{n}\left(X_{1}\right)>t\right\} \leq P\left\{k^{-1 / p} \sum_{i=1}^{k} q_{n}\left(X_{i}\right)>t\right\} .
$$


By $\theta$ and $\theta_{n}$ we denote $p$-stable random variables concentrated on $(0, \infty)$ and such that $q\left(X_{1}\right)$ and $q_{n}\left(X_{1}\right)$ belong to their domains of attraction. From the convergence

$$
P\left\{k^{-1 / p} \sum_{i=1}^{k} q_{n}\left(X_{i}\right)>t\right\} \rightarrow P\left\{\theta_{n}>t\right\} \quad \text { as } k \rightarrow \infty
$$

and from (4) we obtain

$$
P\left\{q_{n}\left(X_{1}\right)>t\right\} \leq P\left\{\theta_{n}>t\right\} .
$$

Now, because

$$
\lim _{n} P\left\{q_{n}\left(X_{1}\right)>t\right\}=P\left\{q\left(X_{1}\right)>t\right\} \quad \text { and } \quad \lim _{n} P\left\{\theta_{n}>t\right\}=P\{\theta>t\},
$$

(5) gives the inequality

$$
P\left\{q\left(X_{1}\right)>t\right\} \leq P\{\theta>t\} .
$$

Now, using in turn (7), (6), (5), (2), and (3) we obtain

$$
\limsup _{t \rightarrow \infty} t^{p} P\left\{q\left(X_{1}\right)>\varepsilon t\right\} \leq \limsup _{t \rightarrow \infty} t^{p} P\{\theta>\varepsilon t\} \leq \nu\{x: q(x)>\varepsilon\}
$$

which completes the proof.

3. In this section we prove our result. To avoid trivial complications we assume throughout the remainder of the paper that the linear span of the set $\{x: q(x) \neq$ $0\} \cap \operatorname{supp} \mu$ is at least two-dimensional. When the above subspace is one-dimensional our theorem obviously remains valid with one exception: at the point 0 there exist only one-sided derivatives of $F$. Denote $U_{t}=\{x: q(x)<t\}$.

THEOREM. Let $E$ be a locally convex separable metric linear space and let $q$ be a lower semicontinuous seminorm on $E$. Let $\mu$ be a symmetric $p$-stable, $0<p<1$, measure on $E$. Assume that $q<\infty \mu$-a.s. and that $\operatorname{dim} \operatorname{lin}(\{q \neq 0\} \cap \operatorname{supp} \mu) \geq 2$. Denote $F(t)=\mu\{x: q(x)<t\}$. Then

(a) the density $F^{\prime}(t)$ exists for all $t \geq 0$ and is continuous on $(0, \infty)$,

(b) the function $f(x)=\mu\left(U_{t}\right)-\mu\left(U_{t}+x\right)$ belongs to $L^{1}(\nu)$, where $\nu$ is the Lévy measure of $\mu$, and for every $t>0$,

$$
F^{\prime}(t)=\frac{p}{t} \int_{E}\left[\mu\left(U_{t}\right)-\mu\left(U_{t}+x\right)\right] d \nu(x),
$$

(c) there exists the limit

$$
\lim _{t \rightarrow \infty} t^{1+p} F^{\prime}(t)=\sigma\left(S_{1}\right)
$$

where $\sigma$ is defined by (1).

The proof is divided into several steps.

Step 1. We prove that under the assumption of the Theorem if $F^{\prime}(s)$ exists then

$$
\frac{s}{p} F^{\prime}(s)=\lim _{t \rightarrow 0+} \frac{1}{t} \int_{E}\left[\mu\left(U_{s}\right)-\mu\left(U_{s}+x\right)\right] d \mu_{t}(x) .
$$


Indeed, if $F^{\prime}(s)$ exists then there exists the following limit:

$$
\begin{aligned}
\lim _{t \rightarrow 0+} \frac{1}{t} \int_{E}\left[\mu\left(U_{s}\right)-\mu\left(U_{s}+x\right)\right] d \mu_{t}(x) & =\lim _{t \rightarrow 0+} \frac{1}{t}\left[\mu\left(U_{s}\right)-\int_{E} \mu\left(U_{s}+x\right) d \mu_{t}(x)\right] \\
& =\lim _{t \rightarrow 0+} \frac{1}{t}\left[\mu_{1}\left(U_{s}\right)-\mu_{1+t}\left(U_{s}\right)\right] \\
& =\lim _{t \rightarrow 0+} \frac{1}{t}\left[F(s)-F\left(\frac{s}{(1+t)^{1 / p}}\right)\right]=\frac{s}{p} F^{\prime}(s) .
\end{aligned}
$$

It is obvious that $F(t)=0$ for $t<0$. Applying our assumption about dimension on the set $\{x: q(x) \neq 0\} \cap \operatorname{supp} \mu$ we infer from Theorem 4.1 in $[1]$ that $F(t)<C t^{2}$ for small $t>0$, hence $F^{\prime}(0)=0$.

Step 2. In this step we prove two important properties of the function $f(x)=$ $\mu\left(U_{s}\right)-\mu\left(U_{s}+x\right)$. Namely, the function $f(x)$ is bounded, continuous $\nu$-a.e., and there exists a constant $M>0$ such that $|f(x)|<M \cdot q(x)$ whenever $q(x)<1$. The function $f$ is clearly bounded and $|f(x)| \leq 1$. If $q(x)<s / 2$ then

$$
f(x)=\mu\left(U_{s}\right)-\mu\left(U_{s}+x\right) \leq F(s)-F(s-q(x))=\int_{s-q(x)}^{s} F^{\prime}(t) d t \leq M_{s / 2} q(x)
$$

because $F^{\prime}(t)<M_{s / 2}$ by virtue of Lemma 1 . Now we show that $f(x)$ is $\nu$-a.e. continuous.

Fix $s>0$ and denote $S_{s}=\{x: q(x)=s\}$. If $x \in E$ is such that $\mu\left(S_{s}+x\right)=0$, then for every sequence $\left(x_{n}\right)$ converging to $x$ we have

$$
\left|f\left(x_{n}\right)-f(x)\right| \leq\left|\mu\left(U_{s}+x_{n}\right)-\mu\left(U_{s}+x\right)\right| \longrightarrow \mu\left(S_{s}+x\right)=0 \text { as } n \rightarrow \infty ;
$$

hence $f$ is continuous at $x$.

We show that $\nu\left\{x: \mu\left(S_{s}+x\right)>0\right\}=0$. Fix $x \in S_{1}$ and let $\mu_{x}$ be a symmetric $p$-stable measure concentrated on $\operatorname{lin}\{x\}$ and with the one-dimensional density $p(y)$. Then $\mu * \mu_{x}$ is also a symmetric $p$-stable measure on $E$ and for every $s>0$ we have $\left(\mu * \mu_{x}\right)\left(S_{s}\right)=0[7]$. Hence

$$
0=\left(\mu * \mu_{x}\right)\left(S_{s}\right)=\int_{-\infty}^{+\infty} \mu\left(S_{s}+y x\right) p(y) d y
$$

and, consequently, for almost all $y \in R$ we have $\mu\left(S_{s}+y x\right)=0$. Let us choose $\varepsilon>0$ and consider a finite measure $\nu_{\varepsilon}=\left.\nu\right|_{\{q>\varepsilon\}}$. We have

$$
\mu * \nu_{\varepsilon}\left(S_{s}\right)=\int_{0}^{\infty} \int_{S_{1}} \mu\left(S_{s}+y z\right) \sigma(d z) \frac{1}{y^{1+p}} d y=0 .
$$

Hence, for $\nu_{\varepsilon}$ almost all $x \in E$ we have $\mu\left(S_{s}+x\right)=0$. When $\varepsilon \rightarrow 0$ we get the desired conclusion.

Step 3 . In this step we show that for every $s>0$

$$
\lim _{t \rightarrow 0+} \frac{1}{t} \int_{E}\left[\mu\left(U_{s}\right)-\mu\left(U_{s}+x\right)\right] d \mu_{t}(x)=\frac{p}{s} \int_{E}\left[\mu\left(U_{s}\right)-\mu\left(U_{s}+x\right)\right] d \nu(x) .
$$

Recall that we denote $f(x)=\mu\left(U_{s}\right)-\mu\left(U_{s}+x\right)$. Let us choose $\varepsilon>0$ and consider the equality

$$
\frac{1}{t} \int_{E} f(x) d \mu_{t}(x)=\frac{1}{t} \int_{\{x: q(x) \leq \varepsilon\}} f(x) d \mu_{t}(x)+\frac{1}{t} \int_{\{x: q(x)>\varepsilon\}} f(x) d \mu_{t}(x) .
$$


Using the well-known estimate $\mu\{x: q(x)>u\} \leq c u^{-p}$ (see [1]) and Step 2 we have

$$
\begin{aligned}
\frac{1}{t} \int_{\{x: q(x) \leq \varepsilon\}} f(x) d \mu_{t}(x) & \leq M_{s} \frac{1}{t} \int_{\{x: q(x) \leq \varepsilon\}} q(x) d \mu_{t}(x) \\
& \leq M_{s} \cdot t^{(1 / p)-1} \int_{\left\{x: q(x) \leq \varepsilon t^{-1 / p}\right\}} q(x) d \mu_{1}(x) \\
& \leq M_{s} \cdot t^{(1 / p)-1} \int_{\varepsilon t^{-1 / p}}^{\infty} \mu\{x: q(x)>u\} d u \leq M_{s}^{\prime} \cdot \varepsilon^{1-p}
\end{aligned}
$$

By virtue of Lemma $\left.2 t^{-1} \mu_{t}\right|_{\{q>\varepsilon\}}$ converges weakly to $\left.\nu\right|_{\{q>\varepsilon\}}$ whenever $\nu\left(S_{\varepsilon}\right)=0$. The function $f(x)$ is $\nu$-a.e. continuous; hence,

$$
\lim _{t \rightarrow 0+} \frac{1}{t} \int_{\{x: q(x)>\varepsilon\}} f(x) d \mu_{t}(x)=\int_{\{x: q(x)>\varepsilon\}} f(x) d \nu(x) .
$$

This ends the proof of Step 3.

Step 4. In this step we show that the function

$$
h(s)=\frac{p}{s} \int_{E}\left[\mu\left(U_{s}\right)-\mu\left(U_{s}+x\right)\right] d \nu(x)
$$

is continuous for all $s>0$. Let us choose and fix $s>0$. Then by virtue of Lemma 1 and Steps 2 and 3 it follows that for every $\varepsilon>0$

$\int_{E}\left[\mu\left(U_{s}\right)-\mu\left(U_{s}+x\right)\right] d \nu(x) \leq \int_{\{x: q(x) \leq \varepsilon\}} M_{s} q(x) d \nu(x)+\int_{\{x: q(x)>\varepsilon\}} 1 d \nu(x)<\infty$.

Now, let $s_{n}$ tend to $s$. Then $\mu\left(U_{s_{n}}\right)-\mu\left(U_{s_{n}}+x\right)$ tends to $\mu\left(U_{s}\right)-\mu\left(U_{s}+x\right)$ for $\nu$-almost all $x \in E$, because, for all $t>0, \mu\left(S_{t}\right)=0$ and $\mu\left(S_{t}+x\right)=0$ for $\nu$-almost all $x \in E$, by Step 2 .

The arguments used in Step 3 imply the convergence $h\left(s_{n}\right)$ to $h(s)$. Hence, the function $h(s)$ is finite and continuous for all $s>0$. By virtue of Step 1 the function $F^{\prime}(s)$ is equal to $h(s)$ for almost all $s>0$. The absolute continuity of $F(s)$ implies that $F^{\prime}(s)$ exists for all $s>0$, it is a continuous function, and the following formula holds:

$$
F^{\prime}(s)=\frac{p}{s} \int_{E}\left[\mu\left(U_{s}\right)-\mu\left(U_{s}+x\right)\right] d \nu(x) \text { for every } s>0 .
$$

Step 5. In this step we show that there exists a constant $C>0$ such that for $s>1$

$$
F^{\prime}(s) \leq C \cdot s^{-1-p}
$$

From Step 4 we know that

$$
F^{\prime}(s)=\frac{p}{s} \int_{E}\left[\mu\left(U_{s}\right)-\mu\left(U_{s}+x\right)\right] d \nu(x) .
$$

We estimate the integral

$$
\begin{aligned}
\int_{E}\left[\mu\left(U_{s}\right)-\mu\left(U_{s}+x\right)\right] d \nu(x) & =\int_{0}^{\infty} \int_{S_{1}}\left[\mu\left(U_{s}\right)-\mu\left(U_{s}+r z\right)\right] \sigma(d z) \frac{1}{r^{1+p}} d r \\
& \leq \sigma\left(S_{1}\right) \cdot\left[\int_{0}^{s} \frac{F(s)-F(s-r)}{r^{1+p}} d r+\int_{s}^{\infty} \frac{F(s)}{r^{1+p}} d r\right] .
\end{aligned}
$$


We estimate both integrals:

$$
\int_{0}^{s} \frac{F(s)-F(s-r)}{r^{1+p}} d r=\lim _{\varepsilon \rightarrow 0} \int_{\varepsilon}^{s} \frac{F(s)-F(s-r)}{r^{1+p}} d r .
$$

Integrating by parts we obtain

$$
\int_{\varepsilon}^{s} \frac{F(s)-F(s-r)}{r^{1+p}} d r=\frac{1}{p} \int_{\varepsilon}^{s} \frac{F^{\prime}(s-r)}{r^{p}} d r-\frac{F(s)}{p s^{p}}+\frac{1}{p} \frac{F(s)-F(s-\varepsilon)}{\varepsilon^{p}} .
$$

Finally,

(8)

$$
\begin{aligned}
\int_{\varepsilon}^{s} \frac{F^{\prime}(s-r)}{r^{p}} d r & =\int_{\varepsilon}^{s / 2} \frac{F^{\prime}(s-r)}{r^{p}} d r+\int_{s / 2}^{s} \frac{F^{\prime}(s-r)}{r^{p}} d r \\
& \leq \max _{s / 2 \leq r \leq s} F^{\prime}(r) \frac{1}{1-p}\left[\left(\frac{s}{2}\right)^{1-p}-\varepsilon^{1-p}\right]+\frac{2^{p}}{s^{p}} \int_{s / 2}^{s} F^{\prime}(s-r) d r \\
& \leq M\left[\left(\frac{s}{2}\right)^{1-p}-\varepsilon^{1-p}\right]+\frac{2^{p}}{s^{p}} \text { by Lemma } 1 .
\end{aligned}
$$

When $\varepsilon \rightarrow 0$ we obtain

$$
\int_{0}^{s} \frac{F(s)-F(s-r)}{r^{1+p}} d r \leq \frac{M}{p}\left(\frac{s}{2}\right)^{1-p}+\frac{2^{p}}{p s^{p}}-\frac{F(s)}{p s^{p}}
$$

The second integral $\int_{s}^{\infty}\left(F(s) / r^{1+p}\right) d r$ is less than

$$
\int_{s}^{\infty} \frac{1}{r^{1+p}} d r=\frac{1}{p s^{p}}
$$

Hence there exists a constant $C_{1}>0$ such that for $s>1$

$$
F^{\prime}(s) \leq \sigma\left(S_{1}\right) \cdot \frac{1}{s} \cdot\left[M\left(\frac{s}{2}\right)^{1-p}+\frac{2^{p}}{s^{p}}\right] \leq C_{1} \cdot s^{-p} .
$$

Let us observe that in (8) we used the estimate $\max _{s / 2 \leq r \leq s} F^{\prime}(r) \leq M$, obtained from Lemma 1 and Step 4. Now we can use the estimate $\max _{s / 2 \leq r \leq s} F^{\prime}(r) \leq$ $C_{1}(s / 2)^{-p} ;$ hence we obtain

$$
F^{\prime}(s) \leq \frac{C_{2}}{s^{2 p}}+\frac{C_{3}^{p}}{s^{1+p}} \quad \text { for } s \text { large enough. }
$$

Iterating this procedure $n$ times, $n=[(p+1) / p]+1$, we obtain: There exists $C>0$ such that $F^{\prime}(s) \leq C s^{-1-p}$ for all $s>1$.

Step 6. In this step we show that $\lim _{s \rightarrow \infty} s^{1+p} F^{\prime}(s)=\sigma\left(S_{1}\right)$. Choose and fix $\varepsilon>0$. For $s$ large enough we have the inequality

$$
\begin{aligned}
F^{\prime}(s) & \geq \frac{p}{s} \int_{\{x: q(x)>(1+\varepsilon) s\}}\left[\mu\left(U_{s}\right)-\mu\left(U_{s}+x\right)\right] d \nu(x) \\
& \geq \frac{p}{s} \int_{\{x: q(x)>(1+\varepsilon) s\}}\left[\mu\left(U_{\varepsilon s}\right)-\mu\left(U_{\varepsilon s}^{c}\right)\right] d \nu(x) \\
& \geq \frac{p}{s} \int_{(1+\varepsilon) s}^{\infty} \sigma\left(S_{1}\right) \frac{F(\varepsilon s / 2)}{r^{1+p}} d r=\frac{F(\varepsilon s / 2) \cdot \sigma\left(S_{1}\right)}{(1+\varepsilon)^{p} \cdot s^{1+p}}
\end{aligned}
$$

hence $\lim \inf _{s \rightarrow \infty} s^{1+p} F^{\prime}(s) \geq \sigma\left(S_{1}\right) /(1+\varepsilon)^{p}$. 
On the other hand we have

$$
\begin{aligned}
\underset{s \rightarrow \infty}{\lim \sup } s^{1+p} F^{\prime}(s) & \\
\leq \underset{s}{\leq} \lim \sup p s^{p} \sigma\left(S_{1}\right) & {\left[\int_{0}^{(1-\varepsilon) s} \frac{K F^{\prime}(\varepsilon s)}{r^{p}} d r\right.} \\
& \left.+\int_{(1-\varepsilon) s}^{s} \frac{F(s)}{r^{1+p}} d r+\int_{s}^{\infty} \frac{F(s)}{r^{1+p}} d r\right],
\end{aligned}
$$

where $K>0$ is a constant. Because $F^{\prime}(\varepsilon s) \leq C /(\varepsilon s)^{1+p}$ by Step 5 , we have

$$
\begin{aligned}
& \underset{s \rightarrow \infty}{\lim \sup p s^{p} \sigma\left(S_{1}\right)} \int_{0}^{(1-\varepsilon) s} \frac{K F^{\prime}(\varepsilon s)}{r^{p}} d r \\
& \quad \leq \limsup _{s \rightarrow \infty} p s^{p} \sigma\left(S_{1}\right) K \frac{C}{(\varepsilon s)^{1+p}} \cdot \frac{1}{(1-p)}[(1-\varepsilon) s]^{1-p}=0 .
\end{aligned}
$$

Estimating the second term of the right-hand side of inequality (10), we get

$$
\begin{aligned}
p s^{p} \sigma\left(S_{1}\right) \int_{(1-\varepsilon) s}^{s} \frac{F(s)}{r^{1+p}} d r & =\sigma\left(S_{1}\right) F(s) s^{p}\left[\frac{1}{s^{p}(1-\varepsilon)^{p}}-\frac{1}{s^{p}}\right] \\
& \leq \sigma\left(S_{1}\right)\left[\frac{1}{(1-\varepsilon)^{p}}-1\right] \quad \text { for every } \varepsilon>0
\end{aligned}
$$

hence this expression tends to 0 as $s \rightarrow \infty$. Estimating the third term in (10) we obtain

$$
\limsup _{s \rightarrow \infty} p s^{p} \cdot \sigma\left(S_{1}\right) \int_{s}^{\infty} \frac{F(s)}{r^{1+p}} d r=\sigma\left(S_{1}\right) .
$$

Finally, $\lim \sup _{s \rightarrow \infty} s^{1+p} F^{\prime}(s)=\sigma\left(S_{1}\right)$, which gives the desired conclusion. This ends the proof of our theorem.

ACKNOWLEDGMENTS. We are very indebted to Mr. M. Ryznar for permitting us to include the proof of Lemma 2. We would like to thank the referee for his valuable remarks.

\section{REFERENCES}

1. A. de Acosta, Stable measures and seminorms, Ann. Probab. 3 (1975), 865-875.

2. T. Byczkowski and K. Samotij, Absolute continuty of stable seminorms, Ann. Probab. 14 (1986), 299-312.

3. V.S. Cirel'son, The density of the distribution of the maximum of a Gaussian process, Theor. Probab. Appl. 20 (1975), 847-855.

4. J. Hoffmann-Jørgensen, L. A. Shepp, and R. M. Dudley, On the lower tail of Gaussian seminorms, Ann. Probab. 7 (1979), 319-324.

5. M. Ryznar, Density of stable seminorms, Bull. Acad. Polon. Sci. Sér. Sci. Math. 33 (1985), 431-440.

6. R. Sztencel, On the lower tail of stable seminorm, Bull. Acad. Polon. Sci. Sér. Sci. Math. 32 (1984), 715-719.

7. T. Żak, On the continusty of the distribution function of a seminorm of stable random vectors, Bull. Acad. Polon. Sci. Sér. Sci. Math. 32 (1984), 519-521.

INSTITUTE OF MATHEMATICS, TECHNICAL UNIVERSiTy, WYBRZEZE WYSPIAŃSKIEGO 27, 50370 WROCŁAW, POLAND 